\title{
Impact of nursing guidelines on nurses' knowledge and performance regarding to prevention of ventilator associated pneumonia in neonates
}

\author{
Safaa Abdel Fattah Abou Zed*1, Amira Adel Mohammed ${ }^{2}$ \\ ${ }^{1}$ Pediatric Nursing Department, Faculty of Nursing, Ain Shams University, Cairo, Egypt \\ ${ }^{2}$ Pediatric Nursing Department, Faculty of Nursing, South Valley University, Qena, Egypt
}

Received: December 4, 2018

Accepted: May 22, 2019

Online Published: July 5, 2019

DOI: $10.5430 /$ jnep.v9n10p15

URL: https://doi.org/10.5430/jnep.v9n10p15

\begin{abstract}
Background: Ventilator Associated Pneumonia (VAP) is a common syndrome in pediatrics primarily in infants and early childhood. Mechanical ventilation is one of the leading supportive modalities of management in the intensive care unit, but it conveys a lot of threats and complications. This study aimed to assess the impact of nursing guidelines on nurses' knowledge and performance regarding to prevention of ventilator associated pneumonia in neonates through the following: 1) Assessment of nurses' knowledge and performance regarding to prevention of ventilator associated pneumonia in neonates. 2) Designing, implementing and evaluating the impact of nursing guidelines on nurses' knowledge and performance regarding to prevention of ventilator associated pneumonia in neonates.

Methods: Research design: A quasi-experimental design was utilized to conduct this study. Settings: The study was convoyed at Maternity \& Gynecological and Children's Hospitals affiliated to Ain shams University Hospitals from neonatal intensive care units. Sample: A convenient sample method of forty three (43) nurses, and fifty (50) neonates' infants on mechanical ventilation was included in the study as a single study group. Tools: The questionnaire format, the observation checklist and implementation of nursing guidelines was assessed the nurses' knowledge and performance regarding to prevention of ventilator associated pneumonia in neonates. The implementation of nursing guidelines was premeditated as reference guidelines for nurses.

Results: There were statistically significant differences between mean scores of the pre and post test as regards nurses' knowledge and performance regarding to prevention of ventilator associated pneumonia in neonates.

Conclusions: Application of the nursing guidelines has a positive outcome on improving nurses' knowledge and performance regarding to prevention of ventilator associated pneumonia in neonates. Recommendations: The current study recommended that directing a written protocol for prevention of ventilator associated pneumonia in neonates to support satisfactory knowledge, and competent practices are actually required at neonatal intensive care units.
\end{abstract}

Key Words: Neonatal intensive care units, Nurses' knowledge, Ventilator Associated Pneumonia (VAP), Performance

\section{INTRODUCTION}

Critically ill neonates who are hospitalized at neonatal intensive care units (NICUs) are at higher threat for several noso- comial infections and respiratory tract infections contributes to a significant proportion of nosocomial infections. ${ }^{[1]}$ Advances in mechanical ventilation (MV) in the neonatal in-

\footnotetext{
* Correspondence: Safaa Abdel Fattah Abou Zed; Email: drsafaa1980@gmail.com; Address: Pediatric Nursing Department, Faculty of Nursing, Ain Shams University, Cairo, Egypt.
}

Published by Sciedu Press 
tensive care units have back to markedly to the survival of neonates infants in the last decades; especially extremely preterm ones. However, Mechanical ventilation is a unique compassionate modalities in the intensive care unit but it conveys a lot of hazards and complications, the ultimate one being ventilator associated pneumonia which can significantly affect the outcome of critically ill neonates. ${ }^{[2]}$

Ventilator-associated pneumonia (VAP) is a most common complication amongst neonates experiencing mechanical ventilation and one of the main forms of health careassociated infection (HAI) related to mechanical ventilation. It considers the second most communal nosocomial infection in NICUs, subsequent great morbidity and mortality. It also extends the length of hospital stay and increase hospital costs. ${ }^{[3]}$ However, any intubated neonates' infant is at threat to increase of VAP rates and extend the duration of mechanical ventilation. Therefore, avoidance of VAP in intubated neonates is thus significance in acute care situations must starts with avoiding or controlling time of mechanical ventilation whenever possible. ${ }^{[4]}$ According to the Center for Disease Control and Prevention (CDC) and National Healthcare Safety Network, VAP is clear as a subtype of pneumonia happens up to 48 hours after beginning of mechanical ventilation in neonates who had no previous lung infections at a period of initiating mechanical ventilation as emplace on the date of occasion or the day before. Inappropriately, little studies have attentive on neonates, particularly those with a low or very low birth weight. ${ }^{[5]}$

The prevalence of VAP in neonate is challenging to be acceptably resolute, because it is hard to discriminate between new or advanced radiographic infiltrates owing to neonatal pneumonia or as a result of exacerbation of broncho-pulmonary dysplasia and recurrent occurrences of atlectasis. Largely, VAP accounting for $6.8 \%$ to $32.2 \%$ of total neonates with nosocomial infection in critical care units and $9 \%-13 \%$ of the total death neonates with ventilator support devices. Although, a universally recognize definition of VAP is still requiring. The rate of 15.7-52 episodes/1,000 ventilation days has been documented in the neonatal population. Consequently, the burden of VAP in NICUs is significantly serious in developing countries than in developed countries correspondingly. VAP is necessary to increase neonates' infant consequences and is an indicator of quality of care. ${ }^{[6,7]}$

Risk factors and consequences of VAP are well-defined in adults; conversely, few data is existent regarding VAP in pediatrics, especially for neonates. Mainly, the major risk factor for increasing VAP in neonates is even complex as a result of their immature immune system, exposed to various invasive medical procedures, increased proportions of multidrug-resistant infections, chronic obstructive pulmonary disease, mechanical ventilation, reintubation, technique of endotracheal suctioning, ventilator circuit changes, persistent neuromuscular block, antibiotics use, opiate action for sedation, gastroesophageal reflux, , subglottal or tracheal stenosis, trauma or surgical problems, prematurity, low birth weight (BW), parenteral nutrition and enteral feeding, prolonged length of stay, and independent risk factors for the development of VAP. ${ }^{[2]}$

VAP diagnosis has confirmed to be routine. Universally recognize measures are yet to be firm. Additionally, the standard diagnostic criteria for VAP as a new and persistent radiographic infiltrates on chest $\mathrm{X}$ ray and deteriorating gas exchange in ventilated neonates for at least 48 hours and who revealed at least 3 of the subsequent criteria: temperature instability (elevation of body temperature $>38^{\circ} \mathrm{C}$ ) with no other familiar cause, leukopenia $4,000 / \mathrm{mL}^{3}$, variation in characteristic of respiratory discharges (purulent pulmonary secretion), rise in nominal fraction of inspired oxygen to $>10 \%$, tachycardia or bradycardia , and respiratory distress. ${ }^{[4,8]}$

Evidence-based strategies for the prevention of VAP and improving the quality of care; ${ }^{[5,9]}$ primarily accomplished by the 'bundle approach'. Which comprises concurrent application of numerous evidence-based preventive approaches that, when implemented together, achieve better patient outcomes. As neonates have different anatomy, physiology, underlying diseases, and they undergo different invasive procedures compare to with older children and adults. Specific studies for evaluating different 'VAP bundles' efficacy in preventing VAP in NICUs is still needed. However, in Egypt and other developing countries, reports on the success of VAP intervention strategies, particularly among neonates, are few. Meanwhile, the bundle included methods to reduce bacterial colonization of the oropharynx, stomach, and sinuses and methods to prevent aspiration of contaminated secretions. VAP rates decreases from 5.6 to 0.3 infections per 1,000 ventilator days once the bundle have implemented during the course of a year. ${ }^{[10,11]}$

Approaches for prevention of VAP in neonates are a challenge when handling the pediatric population. The lacking in evidence to support best practices. These VAP prevention bundles include avoid or decrease duration of mechanical ventilation, minimize ventilator circuit changes. Moreover, use a separate devices for oral \& endotracheal (ETT) suctioning, selection of orotracheal intubation over nasotracheal intubation to decrease sinusitis, checking of gastric residuals to avoid aspiration and inline suctioning, resuscitation bags changed every week and hang up at bedside rather than puts 
it in the bed. In addition, perform routine environmental decontamination with germicidal wipes, avoid heavy sedation $\&$ paralytics to decrease aspiration risk, Prevent ventilator tubing condensate from entering airway, maintain head of the bed (HOB) 30-45 degrees and positioning of neonates must be keep raised at 10-15 degrees, maintenance of bedside, measure intubation readiness daily, and oral hygiene. ${ }^{[12-14]}$

Neonatal nurses at critical care settings play an imperative role in prevention of ventilator-associated pneumonia and risk factors associated. The care bundle built on factors, including evidence concerning the etiology of pneumonia, pathophysiology, and mechanical ventilation, interval of ventilation, and neonates' age, can propose a persistent prevention approaches for care providers caring for neonates at NICUs. The recommendations of The Centers for Disease Control and Prevention and adjusting an adult model also can support this struggle. Finally, neonatal nurse guides care, consuming best evidence to avoid this significant health problem. ${ }^{[15]}$ However, approaches to prevent VAP in neonates including; staff training, transmission precautions, and bundle practices. Staff training, hand washing routine and oral hygiene with antiseptic solution are very significant policies to certify compliance and decrease nosocomial infections. In addition to, airway clearance is important to sustain a patent airway and to manage secretions by use of normal saline instillation (NSI) before endotracheal suctioning has practiced widely at NICUs for soften the thick secretions, and facilitate the removal of secretions. ${ }^{[16]}$

Lack of nurses' knowledge and performance may be a barrier to achieve evidenced based guidelines for preventing ventilator associated pneumonia and transforming evidence based outcomes into regular delivering care is a challenge. Nevertheless, many studies have revealed that, educational programs and implementing nursing guidelines for prevention of ventilator associated pneumonia leading to a significant decrease rates of ventilator associated pneumonia. ${ }^{[17]}$

\subsection{Significance of the study}

Mechanical ventilation considered the most and main supportive management modalities at NICUs; it carries out more hazards and complications. Ventilator associated pneumonia is the most common one which could be considerably affected on the nurses' outcome caring for critically ill neonates with pneumonia. VAP has been associated with great morbidity, mortality, increase length of stay at NICU, and increased hospitalization costs. In developing countries which reported a higher VAP rates compared with the developed countries. Furthermore, studies monitoring VAP rates and success of intervention strategies in Egyptian NICUs are few.

Published by Sciedu Press
Prevention guidelines of Ventilator-associated pneumonia from Center for Disease Control and Prevention (CDC), American Thoracic Society (ATS), and the Institute for Health Care and Improvement (IHI) have been published to decrease the ventilator-associated pneumonia rates in clinical care settings; however, compliance of nurses regarding prevention guidelines is questionable. In addition, VAP has indicated a key challenge to community health, significant health and financial consequences. Moreover, previous studies have reinforced the nurses' knowledge and practices about prevention of ventilator associated pneumonia among adults. But few researches focused on the nurses' knowledge and performance related to prevention of ventilator associated pneumonia at NICUs. In Egypt; there is shortage of staff nursing, expert and knowledgeable nurses are particularly vital and desirable to make proper decisions in neonatal care and minimize risks. Knowledge on evidence based practices should carry self-confidence to intensive care nurses to make appropriate decisions and minimize poor outcomes in the recovery of mechanically ventilated neonates. Consequently, the aim of this study is to assess the impact of nursing guidelines on nurses' knowledge and performance regarding to prevention of ventilator associated pneumonia in neonates.

\subsection{Aim of the study}

This study aimed to assess the impact of nursing guidelines on nurses' knowledge and performance regarding to prevention of ventilator associated pneumonia in neonates. Through: 1) Assessment of nurses' knowledge and performance regarding to prevention of ventilator associated pneumonia in neonates. 2) Designing, implementing and evaluating the impact of nursing guidelines on nurses' knowledge and performance regarding to prevention of ventilator associated pneumonia in neonates.

\subsection{Research hypothesis}

The present study hypothesized that: Implementation of nursing guidelines will improve nurses' knowledge and performance regarding to prevention of ventilator associated pneumonia in neonates positively.

\section{Methods}

\subsection{Research design}

The current study design was a quasi-experimental design where the nurses' sample served as their own control (single group pre-test/post-test design).

\subsection{Setting}

The current study conducted at NICUs at Maternity \& Gynecological Hospital and Children's Hospital affiliated to Ain 
Shams University Hospital. Where the two settings being of the highest capacity of nurses and neonates.

\subsection{Sample and sample size}

A convenient sample was utilized in this study forty three (43) nurses, and fifty (50) neonates' infants on mechanical ventilation were available in the time of data collection were selected according to certain inclusion criteria: such as gender, gestational age, chronological age, birth weight, number of days on MV, type of delivery and presence of congenital anomalies. Practitioner nurses were with various educational categories represented and all should be participated voluntarily in the current study.

\subsection{Tools for data collection}

Three tools were used for data collection:

Tool (I): A predesigned questionnaire sheets (pre/post test), it was developed by the researchers based on. ${ }^{[18,19]}$ It was designed in a simple Arabic language to assess nurses' knowledge and performance regarding to prevention of ventilator associated pneumonia in neonates. These tools were tested for its content validity and reliability by a jury before its implementation. It included three parts as

Part (1): (A) Socio-demographic data for studied nurses which included; nurses' age, qualifications, experience years, and attendance of previous training programs about prevention of VAP in neonates. (B) Socio-demographic data for studied neonates as regards; gender, gestational age, chronological age, birth weight, number of days on MV, type of delivery and presence of congenital anomalies.

Part (2): Assess the nurses' knowledge regarding to prevention of ventilator associated pneumonia in neonates, data collected by used of a questionnaire sheet (pre /post test), it consists of 18 multiple choice and true/false questions. It included; question were covered definition \& causes of VAP, risk factors \& outcomes of VAP, clinical picture, indication of $\mathrm{MV}$, and complications, and management of MV, it also including questions related to nursing care of neonates on MV (before, during, and after) initiation of mechanical ventilation, and questions covered VAP prevention guidelines pre/post nursing implementation.

Scoring system: Each question in questionnaire sheet format was ranged from 5 to 10 grades according to its importance, and weight of it. A pre designed key answers was used, and accordingly answers obtained were checked to be satisfactory or unsatisfactory knowledge. The total score of questionnaire format was 100 grades (equal 100\%).

- Satisfactory knowledge: $\geq(60 \%)$

- Unsatisfactory knowledge: less than $(60 \%)$
Tool (II): Follow up observation checklist (pre/post test), these tool were designed by the researchers based on. ${ }^{[5,9,20,21]}$ The designed observation checklists were used to assess and evaluate the nurses' practices regarding to prevention of Ventilator associated pneumonia pre and post implementation of nursing guidelines. This tool included 8 items which were covered the actual nurses' practices of hand washing, protective clothes, gloves, gowns and mask, mechanical ventilator care \& settings, route of endotracheal intubation, endotracheal suctioning techniques and equipment, enteral feeding, oral hygiene, and neonates' positioning. It was tested for its content validity and reliability.

Scoring system: Follow up observation checklists was developed; the steps of each procedure were distributed as done competent scored (1), and done incompetent scored (0). The total score of checklists were 120 grades. The scoring system for these observation checklists sheets were classified into:

- Competent $\geq 85$ grades.

- Incompetent less than $85 \%$ grades.

Tool (III): Implementation of nursing guidelines program for prevention of ventilator-associated pneumonia. The application of nursing guidelines in the study was guided according to. ${ }^{[5]}$ The evaluation was conducted and the implementation nursing guidelines program were then designed based on the actual needed assessment of the studied nurses through reviewed the related literature. The implementation nursing guidelines program were contained the theoretical and practical skills. The researchers were used different teaching methods during the implementation of nursing guidelines program it included; lectures, group discussion, demonstration and redemonstration and various teaching media such as watching videos, posters, power point presentation and hand-out for prevention of VAP in neonates. In addition, a nurses' guidelines booklet would be designed by the researchers in Arabic language with regard to VAP prevention guidelines protocol. It was considered a nurses' guidelines referral to nurses in neonatal intensive care units. The nursing guidelines booklet was revised for the validity and clarity of three professional in pediatric nursing. In the light of their comments, the researchers were made the necessary adjustments in the final form of the nurses' guidelines manual. Nurses' knowledge and performance were evaluated 2 times pre/and immediate post after implementing nursing guidelines program by using the previously mentioned study tools.

\subsection{Validity and reliability of the study tools}

The study tools were reviewed by five experts in the Pediatric Nursing professional, who reviewed the instruments for clarity, relevance, understanding, comprehensiveness, applicabil- 
ity and easiness. In the light of their revision, modifications in the face validity were carried out.

\subsection{Ethical considerations}

The approval of the research was attained from the research ethics committee affiliated to faculty of nursing before the study was initiated. The pilot study as well as the study was approved from the directors of Maternity \& Gynecological Hospital and Children's Hospital affiliated to Ain Shams University hospitals. Consent informed was taken from each nurse would included in the study sample and that participation was voluntary. At the initial interview was taken a sufficient explanation about the study. All information provided would be kept confidential. The participants were informed that there were no known risks or hazards known would be associated with the participation or non-participations in this study.

\subsection{Pilot study}

A pilot-tested for feasibility, clarity and time required are applied done on $10 \%$ from the sample size were 6 nurses. Nurses of the pilot study were excluded from the study sample finally.

\subsection{Procedure of data collection}

\subsubsection{Implementation phase}

Present study was carried out at the first week of March (2018) up to the first week of September (2018) for data collection and implementation of nursing guidelines program. The researchers were visited the pervious mentioned study settings, three days/week (Saturday, Monday and Thursday) from 10:00 am to 4:00 $\mathrm{pm}$. The researchers were introduced themselves and gave explanation regarding the aim of the study and the expected outcomes were explained for the studied sample, each nurse was interviewed individually after taken their consent to contribute in the study, they enhance cooperatively to participate in the study. All participants were served as single studied group, constituted 43 nurses. The researchers were collected the socio-demographic data, after that was assessed nurses' knowledge's regarding to prevention of VAP in neonates a pre-test distributed for the recruited sample. At this time, the researchers were good observed the nurses during their work and completed the follow up observation checklist. The implementation of nursing guidelines program were premeditated based on the actual nurses' needed assessment of the studied nurses through reviewed the related literature. The implementation of nursing guidelines program were covered the theoretical and practical skills regarding to prevention of VAP in neonates. It was aimed to improve the nurses 'knowledge and performance regarding to prevention of ventilator-associated pneumonia in neonates. Nurses were divided into small groups, the implementing nursing guidelines program were proceed over 12 demonstrated session; six theoretical and six practical sessions (around 45-60 minutes for each) at various morning shifts by moderate of 3 sessions/week. It was given over a period of 8 weeks. The researchers used different teaching methods during implementation of nursing guidelines program it was included; lectures, group discussion, demonstration and redemonstration and various teaching media such as watching videos, posters, power point presentation and hand-out for prevention of VAP in neonates.

\subsubsection{Evaluation phase}

Immediately after ended the implementation of nursing guideline program, nurses' knowledge and performance were evaluated used the same study tools of pre-test, for the posttest that done to evaluate the program effectiveness. After that, the conclusion and recommendations of the study were distributed to the recruited sample, all nursing staff at the NICU from the Maternity \& Gynecological Hospital and Children's Hospital affiliated to Ain Shams University, had copies from the booklets of the implementation nursing guidelines regarding to prevention of VAP in neonates.

\subsection{Data analysis}

The data were analyzed using the statistical package for social science (SPSS) version 21. Quantitative data were presented as mean and standard deviation (SD), $t$-test was used to determine the differences. Qualitative data were presented as percentage (\%). The significance of the observed difference was obtained at $p<.05$.

\section{Results}

Table 1 shows the socio-demographic data of the studied sample. It was found that the mean nurses' age was $26.63 \pm$ 4.25 years old and approximately two thirds of them $(62.7 \%)$ had diploma in nursing (secondary schools). Also, the mean length of experience was $7.31 \pm 3.81$ years. Nearly two thirds $(65 \%)$ had not attended of previous training programs, while only $35 \%$ of nurses' had attended of previous training programs about prevention of VAP in neonates.

As observed from Table 2, about half of the studied neonates' (54\%) of them was their gestational age from 30 to less than 32 weeks and their mean gestational age $(30.8 \pm 2.1)$. Also, more than half $(58 \%)$ of their chronological age were from 20 to more than 30 days with their mean birth weight $1,723.54 \pm 413.5$ grams and mean days on MV was (39.4 \pm 7.38). Although, approximately two thirds of studied neonates $(62.0 \%)$ were delivered by cesarean section and only $16.0 \%$ of them having congenital anomalies. 
Table 1. Socio-demographic data of the studied nurses in NICU

\begin{tabular}{|c|c|c|}
\hline \multirow{2}{*}{ Nurses' Characteristics } & \multicolumn{2}{|c|}{ Total Number $=43$} \\
\hline & No. & $\%$ \\
\hline \multicolumn{3}{|l|}{ Age in years } \\
\hline$<20$ years & 5 & 11.6 \\
\hline $20-<25$ years & 14 & 32.5 \\
\hline $25-<30$ years & 18 & 41.8 \\
\hline $30-<35$ years & 4 & 9.3 \\
\hline$\geq 35$ years & 2 & 4.6 \\
\hline $\bar{x} \pm \mathrm{SD}$ & \multicolumn{2}{|c|}{$26.63 \pm 4.25$} \\
\hline \multicolumn{3}{|l|}{ Level of education } \\
\hline Diploma in nursing (secondary school) & 27 & 62.7 \\
\hline Diploma with specialties & 7 & 16.2 \\
\hline Technical institute of nursing & 5 & 11.6 \\
\hline Bachelor in nursing & 4 & 9.3 \\
\hline \multicolumn{3}{|l|}{ Years of experience } \\
\hline$<5$ years & 20 & 46.5 \\
\hline $5-<10$ years & 11 & 25.5 \\
\hline $10-<15$ years & 7 & 16.4 \\
\hline$\geq 15$ years & 5 & 11.6 \\
\hline $\bar{x} \pm \mathrm{SD}$ & \multicolumn{2}{|c|}{$7.31 \pm 3.81$} \\
\hline \multicolumn{3}{|c|}{$\begin{array}{l}\text { Attendance of previous training courses about prevention of } \\
\text { VAP in neonates }\end{array}$} \\
\hline Yes & 15 & 35 \\
\hline No & 28 & 65 \\
\hline
\end{tabular}

In relation to the differences between mean Scores of nurses' knowledge regarding ventilator associated pneumonia in neonates' pre and post implementation of nursing guidelines, Figure 1 revealed that there were highly statistically significant differences between mean scores of the pre and post knowledge scores regarding definition \& causes of VAP, risk factors \& outcomes, clinical pictures, indications of MV, complications from MV, and management and between the mean scores of nurses' total knowledge Pre and Post implementation of nursing guidelines.

Table 3 represented the mean scores of nurses' knowledge regarding nursing care of neonates on mechanical ventilation pre and post implementation of nursing guidelines. The mean scores regarding to nursing role before, during, and after initiating of mechanical ventilation pre implementation of nursing guidelines were $4.72 \pm 1.03,4.81 \pm 0.96$ and $4.93 \pm$ 0.99 . On the other hand, the mean scores regarding nursing role before, during, and after initiating of mechanical ventilation post implementation of nursing guidelines were 8.81 $\pm 0.88,8.88 \pm 1.05$ and $8.88 \pm 0.88$. That was reflected the implementation of nursing guidelines proficiency on nurses' knowledge as regards prevention of VAP in neonates.

Table 4 revealed that the mean scores of nurses' knowledge regarding to VAP prevention guidelines pre and post nursing implementation. This table was illustrated that, the total mean scores of nurses knowledge pre nursing implementation was $13.53 \pm 5.20$. While, in the other hand, the total mean scores of nurses knowledge post nursing implementation was $36.86 \pm 3.67$ with highly statistically significant differences between mean scores of knowledge pre and post nursing implementation. It was reflected the positive impact of the nursing implementation guidelines on nurses' knowledge regarding VAP prevention in neonates.

Table 2. Distribution of the studied neonates regards to socio-demographic data

\begin{tabular}{lll}
\hline \multirow{2}{*}{ Neonates' characteristics } & \multicolumn{2}{l}{ Total number $=\mathbf{5 0}(\mathbf{1 0 0} \%)$} \\
\cline { 2 - 3 } & No. & $\mathbf{\%}$ \\
\hline Gestational age (weeks) & 5 & 10 \\
$<30$ & 27 & 54 \\
$30-<32$ & 16 & 32 \\
$32-<34$ & 2 & 4 \\
$34-<36$ & &
\end{tabular}

$>36$

$\bar{x} \pm \mathrm{SD}$

$30.8 \pm 2.1$

Gender

Male

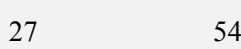

Female

23

46

Chronological age (Days)

$1-<10$

$5 \quad 10$

$10-<20$

$16 \quad 32$

$20-<30$

29

Birth weight (Grams)

$<1,000$

$1,000-<1,500$

$1,500-<2,000$

$4 \quad 8$

$22 \quad 44$

$2,000<2,500 \quad 19 \quad 38$

$\geq 2,500$

$5 \quad 10$

$\bar{x} \pm \mathrm{SD}$

$1,723.54 \pm 413.5$

Number of days on MV (days)

$\begin{array}{lll}15-<30 & 7 & 14 \\ 30-<45 & 18 & 36 \\ 45-\leq 60 & 25 & 50 \\ \bar{x} \pm \text { SD } & 39.4 \pm 7.38 & \end{array}$

\section{Type of delivery}

Cesarean section (CS) $\quad 31 \quad 62$

Normal vaginal delivery (NVD) $\quad 19 \quad 38$

Presence of congenital anomalies

$\begin{array}{lll}\text { Yes } & 7 & 16\end{array}$

$\begin{array}{lll}\text { No } & 36 & 84\end{array}$ 


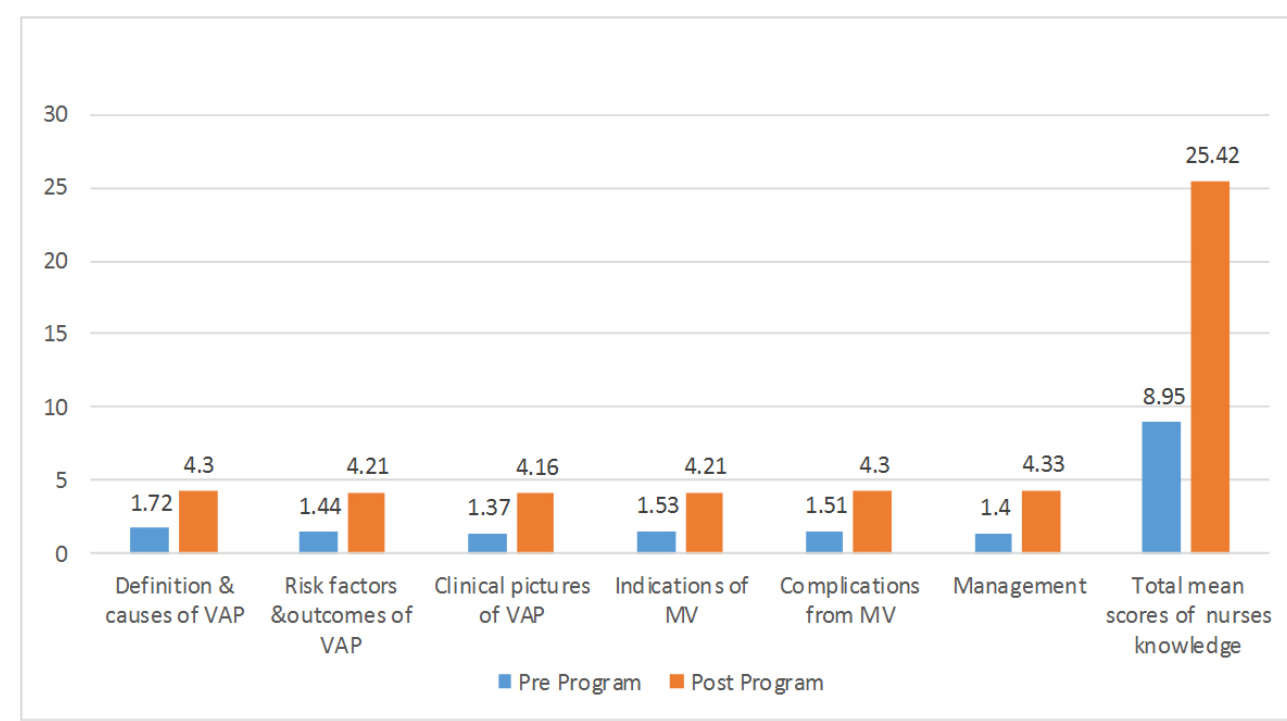

Figure 1. Mean scores of nurses' knowledge regarding Ventilator associated pneumonia in neonates' pre and post implementation of nursing guidelines $(n=43)$

Table 3. Mean scores of nurses' knowledge regarding nursing care of neonates on mechanical ventilation pre and post implementation of nursing guidelines

\begin{tabular}{|c|c|c|c|c|c|}
\hline \multirow[t]{2}{*}{ Knowledge items } & \multirow{2}{*}{$\begin{array}{l}\text { Maximum } \\
\text { score }\end{array}$} & \multirow{2}{*}{$\begin{array}{l}\text { Pre Program } \\
(\mathrm{N}=43) \\
\bar{x} \pm \mathrm{SD}\end{array}$} & \multirow{2}{*}{$\begin{array}{l}\text { Post Program } \\
(\mathrm{N}=43) \\
\bar{x} \pm \mathrm{SD}\end{array}$} & \multirow{2}{*}{$\begin{array}{l}\text { Paired } \\
t \text {-test }\end{array}$} & \multirow[t]{2}{*}{$p$ value } \\
\hline & & & & & \\
\hline Nursing role before initiating of mechanical ventilation & 10 & $4.72 \pm 1.03$ & $8.81 \pm 0.88$ & $-23.752 *$ & .000 \\
\hline Nursing role during initiating of mechanical ventilation & 10 & $4.81 \pm 0.96$ & $8.88 \pm 1.05$ & $-20.853 *$ & .000 \\
\hline Nursing role after initiating of mechanical ventilation & 10 & $4.93 \pm 0.99$ & $8.88 \pm 0.88$ & $-19.284^{*}$ & .000 \\
\hline Total mean scores of nurses knowledge & 30 & $14.47 \pm 2.45$ & $26.58 \pm 2.63$ & $-24.536 *$ & .000 \\
\hline
\end{tabular}

*Significance difference at nurses' knowledge's pre and post-program

Table 4. Mean scores of nurses' knowledge regarding to VAP prevention guidelines pre and post nursing implementation

\begin{tabular}{|c|c|c|c|c|c|}
\hline \multirow[t]{2}{*}{ VAP prevention guidelines items } & \multirow[t]{2}{*}{$\begin{array}{l}\text { Maximum } \\
\text { score }\end{array}$} & $\begin{array}{l}\text { Pre Program } \\
(N=43)\end{array}$ & $\begin{array}{l}\text { Post Program } \\
(\mathrm{N}=43)\end{array}$ & \multirow[t]{2}{*}{$\begin{array}{l}\text { Paired } \\
\text { t-test }\end{array}$} & \multirow[t]{2}{*}{$\begin{array}{l}p \\
\text { value }\end{array}$} \\
\hline & & $\bar{x} \pm$ SD & $\bar{x} \pm$ SD & & \\
\hline $\begin{array}{l}\text { Head of the bed (HOB) maintain at } 30-45 \text { degrees \& } \\
\text { Neonates' positioning must be keep raised at } 10-15 \text { degree }\end{array}$ & 4 & $1.47 \pm 0.70$ & $3.56 \pm 0.55$ & $-17.570 *$ & .000 \\
\hline Hand hygiene & 5 & $1.93 \pm 0.86$ & $4.42 \pm 0.59$ & $-16.567^{*}$ & .000 \\
\hline Oral hygiene with chlorohexidine use and sterile water & 4 & $1.35 \pm 0.61$ & $3.67 \pm 0.57$ & $-20.412 *$ & .000 \\
\hline Orotracheal suctioning vs. nasotracheal intubation route & 5 & $1.63 \pm 0.82$ & $4.49 \pm 0.67$ & $-18.947^{*}$ & .000 \\
\hline $\begin{array}{l}\text { Change ventilator circuit every } 7 \text { days or when circuit visible } \\
\text { soiled or mal functioning }\end{array}$ & 5 & $1.40 \pm 0.82$ & $4.51 \pm 0.67$ & $-19.871 *$ & .000 \\
\hline $\begin{array}{l}\text { Drain condensation from vent circuit every } 2-4 \text { hrs and } \\
\text { before repositioning }\end{array}$ & 4 & $1.49 \pm 0.63$ & $3.60 \pm 0.58$ & $-18.200 *$ & .000 \\
\hline Cuffed ETT tube and cuff pressure checks & 4 & $1.28 \pm 0.67$ & $3.67 \pm 0.52$ & $-21.566 *$ & .000 \\
\hline $\begin{array}{l}\text { ETT tube with extra lumen for drainage of subglottic } \\
\text { secretions }\end{array}$ & 5 & $1.60 \pm 0.82$ & $4.60 \pm 0.66$ & $-19.672 *$ & .000 \\
\hline Frequency of humidifier change. & 4 & $1.35 \pm 0.61$ & $4.51 \pm 0.74$ & $-19.429 *$ & .000 \\
\hline Total mean scores of nurses knowledge & 40 & $13.53 \pm 5.20$ & $36.86 \pm 3.67$ & $-26.329 *$ & .000 \\
\hline Overall total score of knowledge & & $37.19 \pm 11.64$ & $89.07 \pm 8.48$ & $-25.045^{*}$ & .000 \\
\hline
\end{tabular}

*Significance difference at nurses' knowledge's pre and post-program 
As regarded to the level of nurses' total knowledge regarding prevention of VAP pre and post implementation of nursing guidelines. It was cleared that, the researchers were observed improvement regarding the knowledge of the study sample post the implementation of nursing guidelines. The nurses' had satisfactory knowledge only $7 \%$ pre implementation of nursing guidelines. On other hand, the percentage improved to $95.3 \%$ immediately post implementation of nursing guidelines respectively. It was reflected that nursing guidelines program had an improvement on the nurse's total knowledge regarding to prevention of VAP in neonates (see Figure 2).

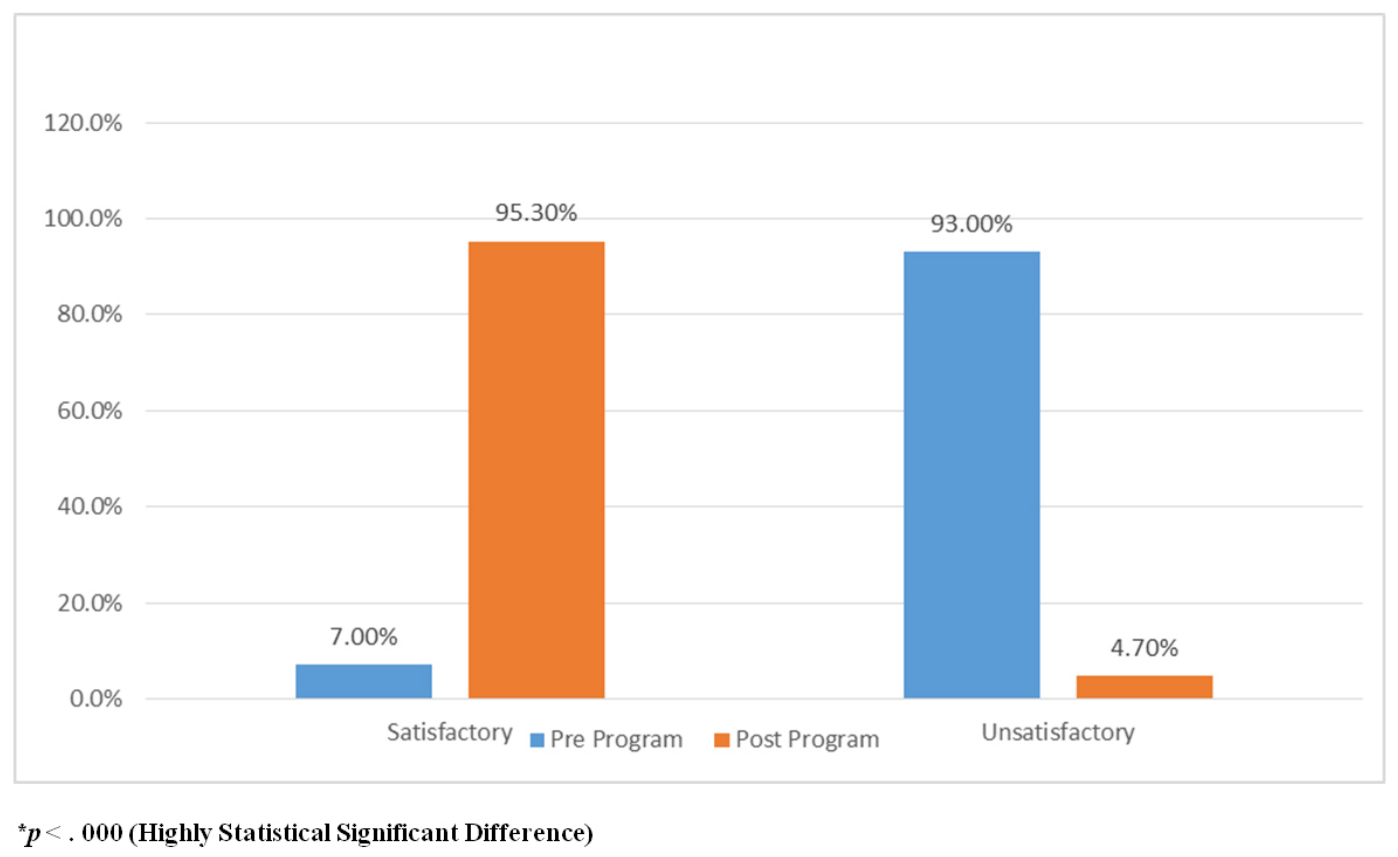

Figure 2. Level of nurses' total knowledge regarding prevention of ventilator associated pneumonia pre and post implementation of nursing guidelines $(n=43)$

Table 5 showed the mean scored of nurses' practice regard- On other hand, immediately post implementation of nursing ing prevention of Ventilator associated pneumonia pre and guidelines the total mean scores was improved to 113.42 post implementation of nursing guidelines. This table was \pm 4.85 respectively. It was reflected the nursing guidelines illustrated that, the total mean score of nurse's practices pre program competency of the nurse's practice regarding to implementation of nursing guidelines was $51.74 \pm 15.68$. prevention of VAP in neonates.

Table 5. Mean scores of Nurses' Practice regarding prevention of Ventilator associated pneumonia pre and post implementation of nursing guidelines

\begin{tabular}{|c|c|c|c|c|c|}
\hline \multirow[b]{2}{*}{ Practice Items } & \multicolumn{5}{|c|}{ Total number=43 (100\%) } \\
\hline & $\begin{array}{l}\text { Maximum } \\
\text { score }\end{array}$ & $\begin{array}{l}\text { Pre Program } \\
\text { Mean } \pm \text { SD }\end{array}$ & $\begin{array}{l}\text { Post Program } \\
\text { Mean } \pm \text { SD }\end{array}$ & $t$-test & $\begin{array}{l}p \\
\text { value }\end{array}$ \\
\hline Hand washing & 15 & $7.16 \pm 2.27$ & $14.05 \pm 0.90$ & $-19.704 *$ & .000 \\
\hline Protective clothes, gloves, gowns and mask & 12 & $6.37 \pm 2.02$ & $11.40 \pm 0.66$ & $-15.868 *$ & .000 \\
\hline Mechanical Ventilator care \& settings & 18 & $6.84 \pm 3.15$ & $16.98 \pm 0.80$ & $-20.656 *$ & .000 \\
\hline Route of Endotracheal intubation & 13 & $5.88 \pm 2.16$ & $12.19 \pm 1.01$ & $-18.263 *$ & .000 \\
\hline Endotracheal suctioning techniques and equipment & 20 & $8.33 \pm 2.35$ & $18.93 \pm 0.94$ & $-26.729 *$ & .000 \\
\hline Enteral feeding & 18 & $6.02 \pm 2.27$ & $17.12 \pm 0.93$ & $-31.384 *$ & .000 \\
\hline Oral hygiene incorporating chlorhexidine use & 12 & $5.81 \pm 2.79$ & $11.33 \pm 0.71$ & $-13.120 *$ & .000 \\
\hline Neonates’ positioning & 12 & $5.33 \pm 2.06$ & $11.44 \pm 0.83$ & $-18.091 *$ & .000 \\
\hline Total mean scores of nurses’ practice & 120 & $51.74 \pm 15.68$ & $113.42 \pm 4.85$ & $-25.593 *$ & .000 \\
\hline
\end{tabular}

*Significance difference at nurses' practice pre and post-program 
As regarded nurses' total practices regarding to prevention of ventilator associated pneumonia pre and post implementation of nursing guidelines. It was observed from Figure 3 that, there was a marked improvement in nurses' total practices immediately after the program implementation with significant statistically differences. Where the majority of nurses (93.0\%) improved in their practice immediately after implementation of the program compared to $7.00 \%$ before implementation of the program respectively.

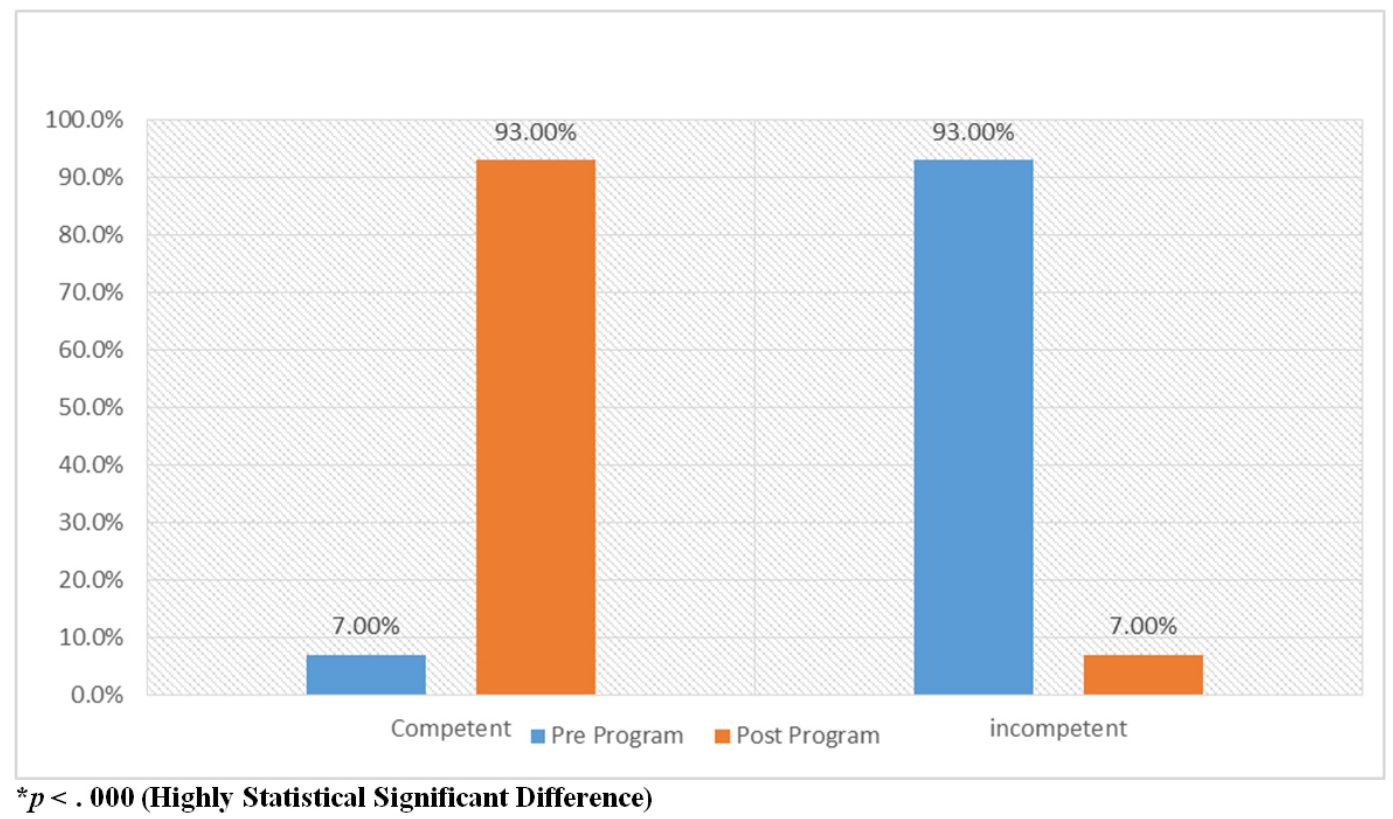

Figure 3. Level of Total Nurses' practice regarding prevention of Ventilator associated pneumonia pre and post implementation of nursing guidelines $(n=43)$

It was cleared from Table 6, implementation of nursing guidelines program enhanced nurses' knowledge and performance regarding prevention of VAP in neonates. It was simplified that, knowledge of suggested guidelines essentially was reflected a proper performance and remained the main step toward the evidence based implementation nursing guidelines for the prevention of VAP in neonates.

Table 6. Correlation between total nurses' total knowledge scores regarding to VAP prevention and total practice scores

\begin{tabular}{|c|c|c|c|c|}
\hline \multirow{2}{*}{$\begin{array}{l}\text { Total } \\
\text { knowledge }\end{array}$} & \multicolumn{2}{|c|}{$\begin{array}{l}\text { Pre-program } \\
(\mathrm{N}=43)\end{array}$} & \multicolumn{2}{|c|}{$\begin{array}{l}\text { Post-program } \\
(\mathrm{N}=43)\end{array}$} \\
\hline & $r$ & $p$-value & $r$ & $p$ value \\
\hline Total practice & $0.945 *$ & $<.001$ & $0.395^{*}$ & .009 \\
\hline
\end{tabular}

\section{Discussion}

VAP is distinct as an emerging pneumonia occurs 48 hour or more after intubation of mechanical ventilation. It is the second most communal nosocomial infection in NICUs. In spite of struggles to prevent VAP, this syndrome continues to occur most recurrently in seriously ill neonates and leading to substantial morbidity and mortality. Prevent and control of nosocomial infections is one of the extreme challenges threatening health care teams all over the world. ${ }^{[17]}$ The present study aimed to assess the impact of nursing guidelines on nurses' knowledge and performance regarding to prevention of ventilator associated pneumonia in neonates.

The current study shown that, the nurses' ages which ranged between 25-30 years (41.8\%) with mean age of $26.63 \pm 4.25$ years represented the highest percentage of the study sample. This result parallel to Metwally ${ }^{[22]}$ whose study about (Effect of Preventive Bundle Guidelines on Nurses' Knowledge and Compliance towards Ventilator Associated Pneumonia in Pediatric Critical Care Units) stated that, in excess of half $(52.9 \%)$ of the studied sample their age ranged between $25-<$ 30 years old with mean age of $26.12 \pm 2.42$ and contrasting with Shahin. ${ }^{[23]}$ We found that three-quarters of the nurses in their study less than 25 years old.

Unfortunately, the results of current study implied that, less than half of the studied nurses (46.5\%) had less than 5 years of experience in NICUs and the majority of them (65\%) didn't attend any previous training programs regarding to prevention of VAP in neonates. This result is in agreement with EL Nabawy ${ }^{[16]}$ who found that the peak percent $(42.9 \%)$ 
were employed in PICU for less than 5 years of experience and the majority of nurses did not attended any training program about prevention of VAP in neonates.

As regards to the socio-demographic characteristics of studied neonates, the results of the present study revealed that, more than half of the studied neonates were males, and their mean gestational age was $30.8 \pm 2.1$ weeks; the neonates mean birth weight was $1,723.54 \pm 413.5$ grams. Also, more than half $(58 \%)$ of their chronological age was from 20 to more than 30 days and mean days on MV was $39.4 \pm$ 7.38. Although, approximately two thirds of studied neonates $(62.0 \%)$ were delivered by cesarean section and only (16.0\%) of them having congenital anomalies. These findings were in contrast with the study done by Khattab et al., ${ }^{[24]}$ stated that, the mean gestational age of infants diagnosed with VAP was significantly lower than that of the non-VAP group. Also, the mean birth weight of the VAP group was significantly lower than that of the non-VAP group $(p=.05)$. Although, prolonged duration of NICU admission was a significant risk factor for VAP. Also, prolonged duration of ventilation generally increases the risk of infection due to exposure to other devices such as nebulizers, humidifiers, and ventilator circuits, which have been proven to be important sources and media for microorganisms. Moreover, the study done by Reem et al. ${ }^{[25]}$ stated that the mean GA and birth weight of the infants diagnosed with VAP were significantly lower than the non-VAP group. That study also showed that premature infants were at a higher risk for developing VAP. This is because of the fact that their need for MV was often for a prolonged interval of time, resulting in a greater number of ventilator days with a steady increase in VAP episodes.

The nurses' institutes were the largest part of employed personnel at hospitals; therefore, any role defect will declines the quality of nursing care given to neonates' infants. Consequently, they need continuous training programs and follow up care delivered to neonates. The aim of nurses' continuous training was to improve knowledge, practice, and attitude of them and eventually to support the quality of nursing care provision to the community. Conversely, a study done by Ibrahim. ${ }^{[26]}$ stressed on training program for nurses as a manner for continuous apprising and restitution of their knowledge and abilities to continue and improve competency. Hence, the aim of the present study was to assess the impact of nursing guidelines on nurses' knowledge and performance regarding to prevention of ventilator associated pneumonia in neonates.

Knowledge is considered the backbone of the prevention of nosocomial infections especially prevention of VAP in neonates. The goal of continuous education in nursing is to improve knowledge and performance to support the quality of care release to pediatrics. ${ }^{[27,28]}$ However, the current study results shown that there were highly statistically significant differences between mean scores of the pre and post implementation of nursing guidelines regarding definition \& causes of VAP, risk factors \& outcomes, clinical pictures , indications of MV, complications from $\mathrm{MV}$, and management and between the mean scores of nurses' total knowledge pre and post implementation of nursing guidelines. On the same line, Ismail. ${ }^{[29]}$ Added that, there were improvements in most items of nurses' general level of knowledge related to VAP post intervention which were statistically significant; however, it has been suggested that, the large number of studied nurses obtained their knowledge from educational training programs, or from hospital strategies and techniques. Furthermore, the participants' demographic data also revealed that, the major part of the studied nurses were secondary school diploma holders. That reflected that nursing guidelines program had an improvement on the nurse's knowledge regarding to prevention of ventilator associated pneumonia in neonates.

Concerning the mean scores of nurses' knowledge regarding nursing care of neonates on mechanical ventilation pre and post implementation of nursing guidelines. The mean scores regarding to nursing role before, during, and after initiating of mechanical ventilation pre implementation of nursing guidelines were $4.72 \pm 1.03,4.81 \pm 0.96$ and $4.93 \pm 0.99$. On the other hand, the mean scores regarding nursing role before, during, and after initiating of mechanical ventilation post implementation of nursing guidelines were $8.81 \pm 0.88$, $8.88 \pm 1.05$ and $8.88 \pm 0.88$. This result was supported from Aloush. ${ }^{[11]}$ Stated that, one reason for nurses' poor compliance might be the lack of education. In this study, $42 \%$ of the nurses reported never having received any education about VAP and $41 \%$ never received education about mechanical ventilator management. This finding supported the report from Sherpa et al. ${ }^{[30]}$ of a significant improvement in nurses' compliance and reduction in the rate of VAP after education in VAP and mechanical ventilator management, even in the presence of a low nurse: patient ratio, which were reflected the implementation of nursing guidelines proficiency on nurses' knowledge as regards prevention of VAP in neonates.

With respect to the mean scores of nurses' knowledge regarding to VAP prevention guidelines pre and post nursing implementation. One of the noticeable findings of this study, the total mean scores of nurses' knowledge pre nursing implementation was $13.53 \pm 5.20$. On the other hand, the total mean scores of nurses knowledge post nursing implementation was $36.86 \pm 3.67$ with highly statistically significant 
differences between mean scores of knowledge pre and post nursing implementation as regards VAP prevention guidelines items such as: neonates' positioning, hand hygiene, orotracheal suctioning vs. naso-tracheal intubation route, change ventilator circuit every 7 days or when circuit visible soiled or mal functioning, drain condensation from vent circuit every 2-4 hours and before repositioning, cuffed ETT tube $\&$ cuff pressure checks, ETT tube with extra lumen for drainage of subglottic secretions, and frequency of humidifier change. On the other hand, the study's results were in agreement with other studies that shown that increase the nurse: patient ratio in NICUs would help to improve their compliance with the guidelines and decrease the rate of VAP. This finding supported those of Bammigatti et al. ${ }^{[31]}$ and Yepez et al. ${ }^{[32]}$ Who reported a significant increase in the risk of VAP in NICUs with a higher workload for nurses; however, future studies are recommended to control for confounding variables in order to attain a better outcomes of the impact of nurses' workload on the rate of VAP in neonates.

In relation to level of nurses' total knowledge regarding prevention of ventilator associated pneumonia pre and post nursing guidelines implementation, the result of current study revealed that, largest number of study sample had unsatisfactory knowledge scores (93\%) pre implementation of nursing guidelines. While, there were marked improvements in nurses' knowledge scores (95.3\%) immediately post implementation of nursing guidelines respectively, in comparison to before implementation of nursing guidelines with highly statistical significant differences. This may be due to no written protocols regarding prevention of Ventilator associated pneumonia in neonates. The knowledge shortage is reflected an obstacles of nursing guidelines implementation for prevention of VAP in neonates as a references available at any times for nurses to be aware of the importance of prevention of VAP and its management. This result concurrent by Gomes. ${ }^{[19]}$ \& Ali. ${ }^{[33]}$ Who convoyed a study about knowledge amongst intensive care nurses on Evidence-based guidelines for the prevention of ventilator-associated pneumonia and their overall knowledge results were poor. Meanwhile, these results may be due to lack of training courses, lack of equipment and work overload which in turn affect nurses' knowledge and practice.

In contrary, the study's results were in contract with Alhirish, ${ }^{[34]}$ El Nabawy et al. ${ }^{[16]}$ who directed a study regarding to evidence based guidelines for prevention of VAP at neonates and their total knowledge that shown poor. These results may be due to lack of training courses, lack of equipment and work overload which in turn affect nurses' knowledge and practice.

Published by Sciedu Press
From the discussion of the participants' mean scores of nurses' practice regarding prevention of Ventilator associated pneumonia pre and post implementation of nursing guidelines. The present study reflected that, the total mean score of nurse's practices pre implementation of nursing guidelines was (51.74 \pm 15.68$)$. On other hand, immediately post-implementation of nursing guidelines the total mean scores was improved to $113.42 \pm 4.85$ in comparison to pre implementation of nursing guidelines respectively with statistical significant differences. In similar study established by Aloush $^{[11]}$ which was conveyed in 10 hospitals in Jordan at NICUs, to assess nurses' knowledge and practices for prevention of ventilator-associated pneumonia through implementation guidelines: It was noticed that the nurses' knowledge and practice was improved post program implementation. It reflects the nursing guidelines program competency of the nurse's practice regarding to prevention of VAP in neonates.

One of the major motive of poor nurses' knowledge and performance is the lack of nurses' education and training. The current study revealed that, $65 \%$ of total studied nurses never received any training programs about prevention of VAP in neonates and mechanical ventilator implementation. The findings supported from Sherpa et al. ${ }^{[30]}$ which reported a significant improvement in nurses' performance and decrease rates of VAP related to mechanical ventilator care management and prevention of its complications. In spite of presence of a low nurse: patient ratio.

With respect to mean score of nurses' practices regarding prevention of Ventilator associated pneumonia pre and post nursing implementation guidelines. In this study, nurses recognized significant reduced in the VAP prevention guidelines items related to do hand washing as they did not pay attention to many steps of hand washing before and after contact with the neonates' infant. Previous studies reported that hand hygiene practices are vital in minimizing the spread of most organisms responsible for VAP, which can reduce the incidence of acute respiratory infections and VAP by up to $50 \%$. The result of the present study might be due to absence of supervision as well as lack of role model may contribute to lack of compliance to hand washing and lack of training program which was recognized by the fact that, the high percentage of the studied nurses did not received any training programs related to infection control. Similarly, Foglia et al. ${ }^{[35]}$ identified that the trainingal involvement and efforts to increase loyalty to hand hygiene for nurses have been associated with decreased VAP rates. In the same line, Ismail ${ }^{[29]}$ Whose study about, the effect of nurses training on VAP prevention bundle on VAP incidence rate at a critical care unit, who reported that, nurses recognized the importance of hand washing as preventive measures of VAP. On 
contrary, significant improvements in nurses' practices were noticed regarding hand washing, shortly post implementation program guidelines. This certified that, there was a positive effect on nurses' practices immediately after program application. In addition, the presence of hand washing technique poster hang up on the NICU wall acts as a reminder for them.

Concerning mean score of nurses' practices regarding prevention of ventilator associated pneumonia pre and post nursing guidelines implementation. The recent study clarify that there were significant statistically differences among studied nurses pre and post nursing implementation guidelines regarding mechanical ventilator care \& setting, in addition to, universal precautions as wear a protective clothes (gown, gloves, and mask). As stated by Khattab et al. ${ }^{[24]}$ that care of the ventilator included: clear the condensate from ventilator circuit and prevent condensate from tiring into the airway of the neonates, heat of ventilator circuits, change ventilator circuits when observed a soiled as preventing contamination of the equipment. This result is concurrent with EL Nabawy et al. ${ }^{[16]}$ who stated that most of the studied nurses confirmed incorrect answer regarding continuous condensation in the ventilator circuit, ventilator circuit changes, maintenance of bed side, air way humidifier, suction system, change of suction systems, types of endotracheal tubes, maintain head of the bed , and hand hygiene.

In the present study, nurses established substantial weakness in performing oral hygiene for neonates on mechanical ventilation by using a Chlorhexidine solution and avoid using of normal saline during suctioning of endotracheal tube. These findings were underlined that training and educational program courses for studied nurses that required to be reflected in design and planning for future enhancement of nurses' performance as regards preventive guidelines of VAP in neonates. These results are in harmony with EL Sayed, et al. ${ }^{[17]}$ who indicated the requisite for standardized oral care protocols in PICUs to improve quality of oral care. Also, it was concluded by Chan \& Hui-Ling. ${ }^{[36]}$ The majority of nurses supposed that careful oral hygiene is vital for neonates on mechanical ventilation. On the other hand, the study supposed that performing oral hygiene to be an unlike task. There was a shortage of standard practice amongst nurses in performing oral hygiene. Performance various with respect to the methods, frequency, and requisites used for oral care.

Moreover, mean score of nurses' practices regarding prevention of Ventilator associated pneumonia pre and post implementation of nursing guidelines. Most of the studied nurses' reported incompetent practices regarding parenteral feeding management in the intended NICUs. The result of the current study was in agreement with Al-Alaiyan et al. ${ }^{[37]}$ who reported that, nurses' practices was unsatisfactory regarding prevention of VAP and parenteral feeding. This might be due to lack of staff nursing to deliver high quality of care for seriously ill neonates. In addition, the nurses' practice was based mainly on past experience and institution, as older nurses teaching procedures to the newly experienced nurses. Evidence-based practices were not a standard care. Consequently, the insufficient practices predisposing neonates' infants to many complications.

Unfortunately, in the current study, the Nurses' Total practices regarding prevention of ventilator associated pneumonia pre and post implementation of nursing guidelines; the findings of the current study implied that, the nurses' total practices pre implementation of nursing guidelines were incompetent (93\%), which oblique threat skills form the nurses. Participants form neonatal intensive care units at mentioned settings there is no suitable documented guideline or another ways which can obtain specific information's to improve their knowledge and performance regarding prevention of Ventilator associated pneumonia in neonates. On other hand, the majority (93\%) of nurses post implementation of nursing guidelines were competent practice. This was a parented in Figure 3 where improvement of nurses' practices and adherence to preventive measures of VAP were recognized. These results were in harmony with Gatell. ${ }^{[38]}$ Whose results showed that, implementation of training program enhanced nurses' knowledge and practices regarding preventive strategies for VAP in neonates.

With reference to the Correlation of nurses' total knowledge and their actual total practices regarding prevention of Ventilator associated pneumonia pre and post implementation of nursing guidelines. The recent study reflected that there were highly statistically difference among nurses' total knowledge and total practices for the study sample pre and post nursing guidelines implementation. This results were expected as it is may be leading to shortage of opportunity for continuous training and education offered in the hospital to enhance the nurses' practice and might be due to also lack of equipment, shortage of staff supervision, as well as shortage of head nurse supervision and close observation and reinforcement as well as the nurses were work overloaded. Meanwhile, the knowledge regarding prevention of Ventilator associated pneumonia is important at the nursing practice.

Finally, continuous training programs and evidence based protocols designed to improve the quality of care for nurses and narrow the cavity between scientific knowledge and actual nurses' performance Gatell. ${ }^{[38]}$ where improvement of nurses' total practice and faithfulness to prevention of VAP in neonates were predictable. This may clarify that, knowledge 
of suggested guidelines essentially reflected proper practices and still it considered the first step toward the implementation of for the prevention of VAP in neonates.

\section{Conclusion}

The study was established that nurses' knowledge and performance regarding prevention of ventilator-associated pneumonia in neonates was improved immediately after the implementation of nursing guidelines than before its implementation. The implementation of nursing guidelines had a positive impact on nurses' knowledge and performance regarding prevention of ventilator-associated pneumonia in neonates at the two previous mentioned settings.

\subsection{Study limitation}

The first limitation of the current study was in its distributed duration, as it doesn't allowed the evaluation of the implementation of nursing guidelines on the long-term period of time, and whether knowledge and performance would remained adequate few months after the training. Also, the recent study didn't included all the factors that would be determined of the occurrence of VAP in neonates; such as the nurses/patients ratio, the shortage of nurses or the workoverload, which sometimes urged the nurses to act in a manner against the procedure steps. Concerned factors would have a vital and important level of determination than possi- ble for this study.

\subsection{Recommendations}

(1) Periodic in-service training program as an essential part of continuous upgrading of nurses' knowledge and performance regarding prevention of ventilatorassociated pneumonia in neonates.

(2) Instituting a system to certify that VAP prevention policies and protocols would be employed instantly in all NICUs and the preventive measures for VAP applied at time of intubation and continue to extubation.

(3) Newly nursing staff orientation at NICUs would including in training and education on policies and strategies for prevention of ventilator-Associated pneumonia in neonates.

(4) Hospital policy should be included as a preventive guideline for the application of VAP prevention protocols in NICU.

(5) Future studies should be conducted to improve nurses' knowledge and performance regarding factors affecting implementation of VAP prevention guidelines in neonates was recommended.

\section{CONFlicts of InTEREST Disclosure}

The authors declare that there is no conflict of interest.

\section{REFERENCES}

[1] Al-Moamary MS, Alhaider SA, Idrees MM, et al. The Saudi initiative for Asthma-2016 update: guidelines for the diagnosis and management of asthma in adults and children. Annals of Thoracic Medicine. 2016; 11: 3-42. PMid:26933455 https ://doi .org/10.4103/18 $17-1737.173196$

[2] Sahlol NY, Madkour LA, Soliman YM. Ventilator associated pneumonia in a tertiary care hospital: incidence, risk factors and etiological agents. British Microbiology Research Journal. 2016; 13: 1-10. https://doi.org/10.9734/BMRJ/2016/24360

[3] Van Oort PM, Nijsen T, Weda H, et al. Breathe Dx-molecular analysis of exhaled breath as a diagnostic test for ventilator-associated pneumonia: protocol for a European multi-Centre observational study. BMC Pulmonary Medicine. 2017; 17: 1-8. PMid:28049457 https://doi.org/10.1186/s12890-016-0353-7

[4] Chreiber MP, Shorr AF. Challenges and opportunities in the treatment of ventilator-associated pneumonia. Expert Review of Anti-Infective Therapy. 2017; 15: 23-32. PMid:27771978 https ://doi .org/10 $.1080 / 14787210.2017 .1250625$

[5] Centers for Disease Control and Prevention (CDC). Pneumonia ventilator-associated (VAP) and non-ventilator-associated pneumonia (PNEU) event. 2016. Available from: http://www.cdc.gov/nhsn /PDFs/pscManual/6pscVAPcurrent

[6] Terjesen CL, Kovaleva J, Ehlers L. Early assessment of the likely cost effectiveness of single-use lexible vide broncho-scopes. Phar- maco Economics-Open. 2017; 1: 133-141. PMid:29442335 https : //doi.org/10.1007/s41669-017-0012-9

[7] Zand F, Zahed L, Mansouri P, et al. The effects of oral rinse with $0.2 \%$ and $2 \%$ chlorhexidine on oropharyngeal colonization and ventilator associated pneumonia in adults' intensive care units. Journal of Critical Care. 2017; 40: 318-322. PMid:28320561 https: //doi.org/10.1016/j.jcrc. 2017.02.029

[8] Roquilly A, Feuillet F, Seguin P, et al. Empiric anti-microbial therapy for ventilator-associated pneumonia after brain injury. The European Respiratory Journal. 2016; 47(4): 1219-1228. PMid:26743488 https://doi.org/10.1183/13993003.01314-2015

[9] IHI. How-to Guide: Prevent Ventilator-Associated Pneumonia. Cambridge: Institute for Healthcare Improvement. 2012.

[10] Akdogan O, Ersoy Y, Kuzucu C, et al. Assessment of the effectiveness of a ventilator associated pneumonia prevention bundle that contains endotracheal tube with subglottic drainage and cuff pressure monitorization. The Brazilian Journal of Infectious Diseases. 2017; 21(3): 276-281. PMid:28193455 https://doi.org/10.1016/j. bjid.2017.01.002

[11] Aloush SM. Nursing students' knowledge about ventilator-associated pneumonia prevention guidelines: effectiveness of a teaching program. American Journal of Infection Control. 2017; 45: 544-546. PMid:28283202 https://doi.org/10.1016/j.ajic.2017.01 .025

Published by Sciedu Press 
[12] Jansen S, Van Der Putten A, Vlaskamp C. What parent find important in the support of a child with profound intellectual and multiple disabilities. Child: Care, Health and Development. 2013; 39: 432-441. PMid:22515566 https : //doi .org/10.1111/j.1365-2214. 20 $12.01381 . \mathrm{x}$

[13] Lerma FÁ, García MS, Lorente L, et al. Guidelines for the prevention of ventilator-associated pneumonia and their implementation. The Spanish “zero-VAP” bundle. medicina Intensiva. 2014; 38: 226-236 https://doi.org/10.1016/j.medine.2013.12.001

[14] Obeid A, Naous A, Naga Z, et al. Preventing ventilator associated pneumonia (VAP) in a Pediatric Intensive Care Unit using a modified ventilator associated pneumonia bundle: interventional and post interventional trial. Research Journal of Medical Sciences. 2014; 8(1): 13-19. https://doi.org/10.5455/ijmr. 20140723095601

[15] Cooper VB, Haut C. Preventing Ventilator- Associated Pneumonia in Children: An Evidence- Based Protocol. Critical Care Nurse. 2013; 33(3): 21-30. PMid:23727849 https://doi.org/10.4037/ccn2 013204

[16] EL Nabawy G, Mustafa O. Knowledge of Pediatric Critical Care Nurses Regarding Evidence Based Guidelines for Prevention of Ventilator Associated Pneumonia (VAP). Journal of Education and Practice. 2015; 6(9): 94-101.

[17] El-Sayed W, El bilgahy A, Hashem S, et al. Effect of implementing training program for nurses' on prevention of pediatric ventilator associated pneumonia. Mansoura Nursing Journal (MNJ). 2015; 2(2).

[18] Blot S, Labeau S, Vandijck D, et al. Evidence based guidelines for the prevention of ventilator-associated pneumonia: results of a knowledge test among intensive care nurses. Intensive Care Med. 2007; 33: 1463-1467. PMid:17541752 https://doi.org/10.1007/s0 0134-007-0705-0

[19] Gomes VA. Knowledge of intensive care nurses on evidence based guidelines for prevention of ventilator associated pneumonia. Master thesis, Faculty of Health Sciences, University of the Witwatersrand. 2010; 145-147.

[20] The American Thoracic Society, Infectious Diseases Society of America. Guidelines for the Management of Adults with Hospitalacquired, ventilator-associated, and healthcare-associated pneumonia American Journal of Respiratory and Critical Care Medicine. 2005; 171: 388-416. PMid:15699079 https://doi.org/10.1164/rc cm. 200405-644ST

[21] World Health Organization. WHO guidelines on hand hygiene in health care: a Summary. First global patient safety challenge clean care is safer care. Geneva. 2009.

[22] Metwally H, El-Sayed W, Shafik F, et al. Effect of Preventive Bundle Guidelines on Nurses' Knowledge and Compliance towards Ventilator Associated Pneumonia in Pediatric Critical Care Units. 4th Annual and 1st International Scientific Conference of Faculty of Nursing Benha University in Collaboration of Psychological Intelligence Foundation (PIFCIC), Hertford, UK. 2015.

[23] Shahin M, Mohamed W. Nurses' knowledge and practices regarding nasogastric feeding at the neonatal intensive care units of Al-Manial University Hospital in Egypt: impact of a Designed Instructional Program. Journal of American Science. 2012; 8(9): 397-404.

[24] Khattab A, El-Lahony D, Soliman W. Ventilator-associated pneumonia in the neonatal intensive care unit. Faculty of Medicine, Menoufia University. 2014; 27: 73-77. https://doi.org/10.4103/1110 $-2098.132753$
[25] Reem M, Tayela A, Abd El Haleem A, et al. Implementation of ventilator associated pneumonia prevention bundle in the neonatal intensive care unit at Alexandria University Children's Hospital, Egypt. Alexandria Journal of Pediatrics. 2017; 30: 74-8. https://doi.org/10.4103/AJOP.AJOP_19_17

[26] El-hanafy E. Impact of Implementation Nursing Guidelines on Minimizing Ventilator Associated Pneumonia among Intensive Care Patients. Journal of Advances in Life Science and Technology. 2015; 29: 40-51.

[27] Malan K. Registered nurses' knowledge of infection control and sterile technique principles in the operating room complex of private hospitals. Master thesis, Faculty of Health Since, Nelson Mandela Metropolitan University. 2009.

[28] Haidegger T, Nagy M, Lehotsky A, et al. Digital imaging for the education of proper surgical hand disinfection. Med Image Comput, Comput Assist Interv. 2011; 14(3): 619-626. https://doi.org/ 10.1007/978-3-642-23626-6_76

[29] Ismail R. the effect of nurses training on ventilator-associated pneumonia (VAP) prevention bundle on VAP incidence rate at a critical care unit. Journal of Nursing Education and Practice. 2015; 5(12): 42-48. https://doi.org/10.5430/jnep.v5n12p42

[30] Sherpa PC, Chakrabarty J, D'SOUZA PJJ. Critical care provider's practices for prevention of ventilator associated pneumonia. Indian Journal of Applied Research. 2016; 5: 337-339.

[31] Bammigatti C, Doradla S, Belgode HN, et al. Health care associated infections in a resource limited setting. Journal of Clinical and Diagnostic Research. 2017; 11: OC01-OC10.

[32] Yepez ES, Bovera MM, Rosenthal VD, et al. Device-associated infection rates, mortality, length of stay and bacterial resistance in intensive care units in Ecuador: international nosocomial infection control Consortium's findings. World Journal of Biological Chemistry. 2017; 8: 95-101. PMid:28289522 https://doi.org/10.4 331/wjbc.v8.i1.95

[33] Ali N. Critical care nurses' knowledge and compliance with ventilator associated pneumonia bundle at Cairo university hospitals. Journal of Education and Practice. 2013; 4(15): 66-77.

[34] Alhirish MH. critical care nurses' application of evidence-based guidelines for preventing ventilator associated pneumonia. MD. Thesis in Critical Care and Emergency Nursing, Faculty of nursing, Alexandria University. 2010.

[35] Foglia E, Meier M, Edward A. Ventilator-associated pneumonia in neonatal and pediatric intensive care unit patients. Clin Microbial Rev. 20:409-425.journal of Neuroscience Nursing. 2007; 40(5): 291-8. PMid:17630332 https://doi .org/10.1128/CMR.00041-06

[36] Chan E, Hui-Ling I. Oral care practices among critical care nurses in Singapore: a questionnaire survey. Applied Nursing Research. 2012; 25(3): 197-204. PMid:21439790 https://doi.org/10.1016/j . apnr. 2010.12.002

[37] Al-Alaiyan S, Binmanee A. Neonatal Ventilator-Associated Pneumonia: An Underdiagnosed Problem in the Neonatal Intensive Care Units. J Pediatr Neonatal Care. 2017; 7(3): 1-5. https: //doi.org/10.15406/jpnc.2017.07.00288

[38] Gatell M, Roig S, Vian O, et al. Assessment of a training programme for the prevention of ventilator-associated pneumonia. British Association of Critical Care Nurses. 2012; 17(6): 285-92. PMid:23061618 https://doi.org/10.1111/j.1478-5153.2012.00526.x 